\title{
LA DEMOCRACIA VENEZOLANA EN UNA ENCRUCIJADA: LAS ELECCIONES NACIONALES Y REGIONALES DE $1998^{1}$
}

\author{
José Enrique Molina V. y Carmen Pérez Baralt*
}

En 1998 el electorado venezolano fue llamado dos veces a las urnas de votación. La primera, el 8 de noviembre para elegir los miembros de las dos cámaras del Congreso $\mathrm{Na}$ cional (diputados y senadores), así como las autoridades de los estados: gobernadores y diputados a las asambleas legislativas. La segunda, el 6 de diciembre para elegir el presidente de la república. Lo ocurrido en estas dos fechas ha cambiado radicalmente el panorama político venezolano, acentuando un proceso que desde el punto de vista electoral se inició en 1993, pero que tiene sus raíces en la crisis social y económica producida por la prolongada caída del precio del petróleo a partir del comienzo de los años ochenta, y en la crisis política que revelaron los motines populares de 1989 y los dos fallidos intentos de golpes de estado de 1992. El principal protagonista de la rebelión militar del 4 de febrero de 1992, Teniente Coronel Hugo Chávez Frías, es ahora elegido Presidente de la República. Por primera vez desde 1958 las organizaciones políticas firmantes del Pacto de Punto $\mathrm{Fijo}^{2}$, o sus derivados, quedan fuera del gobierno y sin mayoría propia en el Congreso. La izquierda, antes excluida de este pacto fundador de la democracia venezolana, y los militares protagonistas de los intentos de golpe de estado de 1992 llegan ahora al gobierno, con una fuerza parlamentaria importante aunque no mayoritaria, y una propuesta de "revolución democrática" (Movimiento V República, 1998: 8), cuyo emblema es la convocatoria a una asamblea constituyente para "refundar la República" (Movimiento V República, 1998: 11). A fin de exponer y explicar lo ocurrido analizaremos en este trabajo, en primer lugar los antecedentes políticos y económicos de las elecciones; en segundo lugar los cambios en el sistema electoral y sus consecuencias; en tercer lugar los partidos políticos y sus candidatos; en cuarto lugar la abstención; en quinto lugar los resultados de las elecciones para el Congreso de la República, gobernadores y cuerpos legislativos de los estados; en sexto lugar la elección presidencial; para concluir con algunas consideraciones sobre las perspectivas futuras de la democracia venezolana.

\section{ANTECEDENTES}

\section{Antecedentes de orden político}

Desde los años cuarenta, al surgir los primeros partidos políticos modernos, una parte importante de los vene- zolanos desarrolló identidades partidistas, que llegaron a su punto más alto en la década de los setenta con el advenimiento del bipartidismo protagonizado por Acción Democrática y COPEI. Estos dos partidos, y en menor medida el Movimiento Al Socialismo (MAS), lograron establecer fuertes lealtades en la población ${ }^{3}$. Para noviembre de 1973, según la encuesta nacional dirigida por Baloyra y Martz (1979), el porcentaje acumulado de militantes y simpatizantes de estos tres partidos alcanzaba $45.9 \%$ (véase Cuadro 1). Para una parte muy significativa de la población la decisión de votar era relativamente sencilla, consistía en apoyar al partido al cual se sentían afectivamente vinculados, y que asumían estaba identificado con sus intereses. Los "electores flotantes" (Pérez, 1992), que resultaban decisivos, también giraban en torno a las opciones representadas por el partido de gobierno o el principal partido de oposición. La crisis económica que comienza en 1982 y se prolonga hasta hoy (nos referiremos a ella más adelante), los escándalos de corrupción en torno a los gobiernos de los dos partidos principales, y la incapacidad de encontrar salidas satisfactorias a los problemas del país, por resumir apresuradamente un proceso complejo, van produciendo una progresiva erosión de estas lealtades, como puede apreciarse en el Cuadro I.

Profesores de Ciencia Política de la Universidad del Zulia.

1. Este trabajo forma parte de un proyecto de investigación financiado por el Consejo Nacional de Investigaciones Científicas de Venezuela, y el Consejo de Desarrollo Científico y Humanístico de la Universidad del Zulia. Organismos a los que agradecemos su apoyo.

2. El Pacto de Punto Fijo fue un acuerdo entre Acción Democrática, COPEI y Unión Republicana Democrática en 1958, con el apoyo de los factores sociales de poder y excluyendo al Partido Comunista, mediante el cual se comprometían a conciliar para el sostenimiento de la democracia, y a cooperar con el gobierno resultante de las elecciones de 1958 mediante un programa común y una coalición parlamentaria y ejecutiva. Más allá de sus efectos inmediatos, ha servido como símbolo de la política de consenso, sobre la cual se estructuró desde sus inicios el sistema democrático.

3. El Cuadro I presenta la evolución de las lealtades partidistas hacia estos tres partidos. Consideramos que ello indica el nivel de la identificación partidista consolidada para cada elección. Hay electores que declaran ser simpatizantes o militantes de otros partidos, particularmente los fenómenos electorales de cada elección en 1993 y 1998, pero éstas no pueden considerarse identidades partidistas consolidadas en el sentido que se analiza aquí. 
Cuadro I

EVOLUCIÓN DE LAS LEALTADES PARTIDISTAS HACIA LOS PARTIDOS TRADICIONALES (AD, COPEI, MAS)

Militantes y simpatizantes de AD, COPEI y el MAS como porcentaje del total de electores

\begin{tabular}{lccccc}
\hline & 1973 & 1983 & 1993 (a) & 1994 & 1998 \\
Militantes & $45.9 \%$ & $35.3 \%$ & $27.8 \%$ & $11.8 \%$ & $14.0 \%$ \\
$\begin{array}{l}\text { Simpatizantes de } \\
\text { AD, COPEI, MAS }\end{array}$ & $(696)$ & $(628)$ & $(398)$ & $(173)$ & $(205)$ \\
\hline Casos Válidos & 1517 & 1778 & 1435 & 1466 & 1463 \\
Casos No Válidos & 4 & 11 & 64 & 34 & 37 \\
Total Casos & 1521 & 1789 & 1499 & 1500 & 1500 \\
\hline
\end{tabular}

(a) Las encuestas de 1973, 1983 y 1998 preguntan si el entrevistado se consi dera militante, simpatizante, independiente o no interesado. En 1993 se hicieron tres preguntas distintas: una define al entrevistado como identificado con algún partido o independiente, otra clasifica a los identificados con un partido en militantes o simpatizantes, y finalmente otra clasifica a todos los entrevistados en muy interesados, interesados y sin interés en política. Para asegurar la comparabilidad se creó una variable con las siguientes categorías: 1) militantes/simpatizantes: incluye a quienes se declararon militantes y a los simpatizantes interesados en política; 2) independientes: incluye a quienes se declararon independientes; 3) no interesados: incluye a los simpatizantes no interesados en política. Creemos que de esta manera se hacen comparable los resultados de 1993 con los de las otras encuestas, aunque al mantener como independientes a los independientes que declararon no tener interés en política, probablemente sobrestime los independientes y subestime los no interesados.

Fuentes: Encuesta Baloyra 73 (Baloyra y Martz, 1979), Encuesta Batoba 83 (Torres, 1985), Encuesta CIEPA/DOXA 1993, Encuesta Red Interuniversitaria de Cultura Política/ DATOS. Las dos primeras fueron facilitadas por el Banco de Datos Latinoamericano de la Universidad Simón Bolívar; Caracas - Venezuela, y las dos últimas por el Instituto de Estudios Políticos y Derecho Público de la Universidad del Zulia.

Como consecuencia de este proceso de erosión de las lealtades, o desalineación partidista (Dalton y Wattenberg, 1993: 205; Särlvik y Crewe, 1983: 332), la volatilidad del electorado se ha incrementado notablemente y la personalidad de los candidatos ha adquirido centralidad con respecto a la decisión de votar (Hidalgo, 1998). Por volatilidad entenderemos la propensión de los electores a modificar su voto entre una elección y otra. El indicador de volatilidad que utilizaremos es el desarrollado por Pedersen (1983) que expresa la variación total de la votación de los partidos entre una elección y otra, es decir el porcentaje de votos que ganan unos partidos y pierden otros desde la elección anterior ${ }^{4}$. En las últimas elecciones con resultado bipartidista, las de 1988, la volatilidad para el voto a la Cámara de Diputados fue de 8\%, en 1993 aumentó a 32\%. En cuanto a la personalización de la política, esta implica que una porción cada vez más importante del electorado toma su decisión de voto con base en una evaluación de la actuación y capacidades de los candidatos para los cargos unipersonales en juego, especialmente gobernadores y presidente. Mientras que la identificación partidista no sólo ha perdido peso, sino que hoy canaliza una porción minoritaria de los sufragios. Estas dos nuevas características del comportamiento electoral venezolano, volatilidad y personalización de la política son un antecedente importante del proceso electoral de 1998, en el cual ambas se acentúan con relación a las elecciones de 1993, primeros comicios nacionales en los que se manifestaron.

Otra consecuencia relevante de la erosión de las lealtades partidistas es el aumento de la importancia de las campañas electorales. Dado el crecimiento de la volatilidad del electorado es de esperar que éste sea ahora más permeable que antes a lo que ocurra en los meses previos a las elecciones, y que la campaña tenga incidencia significativa en el resultado (Alvarez, 1994).

Las elecciones previas, tanto las nacionales de 1993 (Baloyra, 1993; Molina y Pérez, 1994) como las regionales y locales de 1995 (Molina y Pérez, 1995; Maingón y Patruyo, 1996) constituyen antecedentes que contribuyen a entender lo ocurrido en 1998. Las elecciones de 1993 marcan un punto de ruptura con el esquema político consolidado a partir de 1973. Se rompe el sistema bipartidista atenuado que tenía a Acción Democrática y COPEI como actores hegemónicos, y al MAS como una tercera fuerza muy minoritaria, para dar comienzo a una situación de multipartidismo inestable con cinco partidos significativos tanto a nivel electoral como parlamentario (Molina y Pérez, 1994). El elemento más característico y premonitorio de este cambio es que el presidente, por primera vez en 1993, no fue candidato ni de Acción Democrática ni de COPEI. El electorado se pronunció por un cambio político, y si bien escogió a una figura tradicional, también es cierto que éste se presentó y fue aceptado como un factor de transformación y ruptura. Las elecciones regionales de 1995, aunque dieron la impresión de que Acción Democrática estaba en condiciones de recuperarse, ratificaron la situación de multipartidismo inestable, es decir propenso a cambios bruscos en la correlación de fuerzas, así como a la aparición y desaparición de actores entre una elección y otra (Molina y Pérez, 1995).

La crisis política que tuvo sus momentos culminantes con la revuelta popular de febrero de 1989 y los fallidos intentos de golpes de estado en febrero y noviembre de 1992, es también un precedente importante para entender lo ocurrido en 1998. En febrero de 1989, el gobierno de Carlos Andrés Pérez, a menos de un mes de su toma de posesión, aumenta el precio de importantes servicios públicos dentro del marco de un programa de ajuste económico neo-liberal. Sus electores, con base en la campaña electoral, esperaban políticas dirigidas a producir de inmediato mayor bienestar a los sectores populares. Como consecuencia se produjeron motines y saqueos de grandes proporciones, con un número elevado de muertos, heridos y bienes destruidos. Un primer e importante signo de que se estaba generando un descontento masivo y radical. En 1992, los intentos de golpe de estado no despiertan apoyo activo de la población, pero sus líderes, derrotados entonces, entre ellos el candidato ganador de la presidencia de la república en 1998, generan un alto nivel de simpatía, en lo que se ha dado en llamar la paradoja de la democracia venezolana. En todas las encuestas, la mayoría de los entrevistados manifestaba al mismo tiempo apoyo a la democracia y simpatía por los militares rebeldes. Un nuevo y dramático signo del descontento y rechazo a la política tradicional y sus actores.

Otro proceso importante para entender los cambios de comportamiento electoral, que además se desarrolló en paralelo a la crisis de legitimidad institucional señalada en el párrafo anterior, es el proceso de descentralización iniciado

4. Se calcula sumando las diferencias en el porcentaje de votos de todos los partidos entre una elección y otra, y dividiendo el resultado entre dos. 
en 1989 con la elección directa de gobernadores y alcaldes, así como con la entrada en vigencia ese mismo año de la Ley de Descentralización, Delimitación y Transferencia de Competencias del Poder Público que amplió las facultades de los entes descentralizados. La descentralización generó el surgimiento de un nuevo liderazgo político, bastante autónomo de las burocracias partidistas, dado que recibía apoyo popular directo, con lo cual se alimentó en forma importante el proceso de personalización de la política. Ya en 1993 tres de los candidatos presidenciales con opción debían su apoyo popular a su gestión como gobernadores o alcaldes 5 . En 1998 dos de las principales opciones tenían este mismo origen. Más aún, una de las lecturas plausibles de la elección presidencial de 1998 es la que ve en ella la confrontación de opciones que representan las dos alternativas para la transformación, abiertas en forma paralela en Venezuela por la reforma del estado y por la crisis política: la primera de procedencia institucional surgida del proceso de descentralización, presentando un liderazgo político de corte gerencial y generando una expectativa de cambio gradual, encarnada por uno de los gobernadores más exitosos: Henrique Salas Römer. La segunda de origen anti-institucional, con un liderazgo político carismático creado a raíz del intento de golpe de estado del 4 de febrero de 1994 y una oferta de cambio revolucionario, representada por el jefe del movimiento insurreccional: Hugo Chávez Frías.

\section{Antecedentes de orden económico}

Este gobierno tenía como antecedente el deterioro de la situación económica y social del país que con algunos altibajos se venía arrastrando desde comienzos de la década de los ochenta, como consecuencia del enorme peso de la deuda externa sobre el presupuesto del Estado y la caída de los precios del petróleo. Así como el intento fallido durante el gobierno de Carlos Andrés Pérez (1989-1993) de aplicar una política de ajustes macro-económicos y apertura, en acuerdo con el Fondo Monetario Internacional. De esa gestión recibió igualmente como herencia una crisis del sistema financiero que llevó a la quiebra a varios de los bancos más importantes, y obligó al gobierno a destinarle una enorme suma de recursos, desviándolos de sus programas originales, acentuando la inflación y la debilidad de la moneda. Luego de vacilaciones que duraron dos años, el gobierno logra generar un clima de consenso que permite la aplicación, casi sin oposición, de una nueva política de ajustes económicos y apertura, que pareció durante dos años darle un rumbo cierto a la economía y abrir un camino de avance. Una nueva caída de los precios del petróleo en 1998 acabó con el clima de optimismo, acentuó la crisis social, la desigualdad y la pobreza abriendo cauce a la radicalización de las exigencias de un nuevo cambio político. No debe olvidarse que para muchos de sus electores la tarea central del gobierno era la de desarrollar una política social y económica alternativa frente al llamado "paquete neo-liberal" del gobierno de Pérez. Esta fue, por lo demás, una de sus ofertas electorales de punta, junto con la de restablecer la estabilidad política.

Los indicadores de inflación, desempleo y pobreza sufrieron un deterioro con relación al período gubernamental anterior, cuando ya estaban en rojo. La inflación promedio para el período $1994-1998$ fue de $59.6 \%$, frente a $45.9 \%$ del quinquenio anterior ${ }^{6}$. Es cierto que en el último año la inflación estuvo cerca de las predicciones oficiales y su nivel fue el más bajo desde 1986, pero aún así continuó siendo elevada al colocarse en 30\%. El desempleo en 1998 alcanzó 11\%, superior al $8.2 \%$ registrado para 1993. El promedio para el período 1994-1998 fue de 11\%, también peor que el de los cinco años anteriores que se situó en $8.9 \%^{7}$. En cuanto a los niveles de pobreza los indicadores disponibles apuntan a un crecimiento notable. Cifras provisionales emitidas por la Oficina Central de Estadística e Informática, utilizando la metodología "Líneas de Pobreza", señalan que la misma creció del $30.7 \%$ en el primer semestre de 1995 al $68.7 \%$ en el primer semestre de 1997. En cuanto a la desigualdad, las cifras suministradas por Baptista (1997) señalan que desde finales de los setenta hasta 1995 se ha mantenido aproximadamente una situación según la cual el $90 \%$ más pobre de la población recibe el $35 \%$ del ingreso nacional, mientras que el 10\% más rico recibe el $65 \%$ del ingreso. Según el mismo autor, el Coeficiente Gini de pobreza se ha mantenido estable en el mismo período alrededor del 0.58. Estos datos sugieren que en el campo de la lucha contra la desigualdad social extrema no ha habido progreso. Así pues en lo que concierne a lo económico y social, se llega a la elección de 1998 con una clara situación de deterioro en algunos aspectos y estancamiento en otros. No es entonces de extrañar que un electorado que buscó en 1993 el mejoramiento económico mediante un cambio político relativamente moderado, se alejara aún más de los factores tradicionales de gobierno.

\section{EL SISTEMA ELECTORAL}

A partir de 1988 la Ley Orgánica del Sufragio ha sido modificada antes de cada elección nacional o regional. Esta vez no hubo excepción. El objetivo central de la nueva Ley Orgánica del Sufragio y Participación Política fue la "despartidización" de los organismos electorales y la automatización del proceso de votación. Con este objetivo se adoptó el principio de la integración total de los organismos electorales por independientes, y no por representantes de partidos políticos como abierta o encubiertamente ocurría hasta ahora. Como signo del cambio se modificó el nombre del máximo organismo electoral que se denomina ahora Consejo Nacional Electoral, sus siete miembros fueron designados por el Congreso en sesión conjunta de las Cámaras, mediante una mayoría calificada de las dos terceras partes de sus miembros. Tan importante como la letra de la Ley fue que los partidos realmente aceptaron nombrar verdaderos independientes, y no los "pseudo" a que estaban acostumbrados.

5. Oswaldo Alvarez Paz (Gobernador del Estado Zulia, 1989-1993), Andrés Velásquez (Gobernador del Estado Bolívar, 1989-1995) y Claudio Fermín (Alcalde de Caracas, 1989-1992).

6. Los datos sobre inflación son los emitidos por el Banco Central de Venezuela.

7. La fuente de los datos sobre desempleo es la Oficina Central de Estadística e Informática, Encuesta de Hogares por Muestreo. 
El resto de los integrantes de los organismos electorales, incluyendo las mesas receptoras de votos, fue designado mediante sorteo. La automatización consistió en el uso de máquinas escrutadoras, lectoras ópticas, que recibieron las boletas directamente del elector, las escrutaron, elaboraron las actas, y en un alto porcentaje comunicaron directamente los resultados vía modem a las juntas regionales electorales y el Consejo Nacional Electoral. La despartidización y la automatización se combinaron para generar consenso en torno a que estas elecciones fueron realizadas con eficacia y limpieza. Esto es un logro político muy importante, ya que la credibilidad de la población en la limpieza de las elecciones había descendido muy peligrosamente para la democracia, como se desprende de la respuesta en 1995 de los entrevistados venezolanos a la siguiente pregunta de la encuesta Latinobarómetro $95^{8}$ : ¿Ud. cree, en términos generales, que las elecciones en este país son limpias o fraudulentas?. La encuesta se administró a 1200 personas, el $72 \%$ respondió que las elecciones en Venezuela son fraudulentas, el $18 \%$ que son limpias, y el $10 \%$ dijo no saber o no respondió. Ante una pregunta similar realizada luego de las elecciones parlamentarias de noviembre de 1998, se notó claramente un cambio positivo, para el $57.7 \%$ las elecciones fueron limpias, para el $39.2 \%$ fraudulentas, y el $3.1 \%$ dijo no saber o no contestó ${ }^{9}$.

Otra modificación con efectos políticos significativos fue la separación de las elecciones presidenciales de las parlamentarias. Desde 1958 hasta 1993 el presidente era elegido en la misma fecha que el Congreso. En 1998 las dos Cámaras del Congreso se eligieron conjuntamente con los gobernadores y las asambleas legislativas de los estados el 8 de noviembre, mientras que el presidente se eligió el 6 de diciembre. Esta reforma, impulsada por Acción Democrática y COPEI, tenía como objetivo reducir el llamado "efecto portaaviones", mediante el cual el voto presidencial atrae al voto parlamentario. Ninguno de los dos partidos tenía opción de triunfo con sus candidatos presidenciales, y asumían, en una sorprendente aplicación de los hallazgos de la Ciencia Política (Shugart y Carey, 1992; Mainwaring y Shugart, 1997), que la separación, aunque parcial, podría aumentar su cosecha de escaños parlamentarios, al hacerla menos dependiente del voto presidencial. Como en efecto ocurrió. La consecuencia negativa de separar las elecciones es que se perjudica la gobernabilidad al reducir el nivel de apoyo parlamentario del presidente, y hacer más difícil para el ejecutivo alcanzar una mayoría propia o en coalición, permanente o para medidas puntuales.

Un tercer cambio de consecuencias importantes fue el relativo al porcentaje mínimo de votos que un partido debe alcanzar para obtener un cargo adicional compensatorio, y asegurar así al menos un escaño en alguna de las Cámaras. La Ley anterior exigía un cociente nacional completo ${ }^{10}$. Ello hubiera sido equivalente en 1998 a 2.08\% para el Senado y $0.53 \%$ para diputados. La nueva Ley Orgánica del Sufragio redujo este umbral de representación a medio cociente, es decir la mitad de los valores arriba indicados. La consecuencia como era de esperarse, fue un aumento notable del número de partidos con representación, y un nuevo factor de incremento del fraccionamiento del Congreso que se suma al efecto producido por la separación de las elecciones para hacer más difícil la construcción de mayorías parlamentarias, y por ende la gobernabilidad.

Otra innovación importante fue la inclusión en Venezuela de una cuota de participación femenina del $30 \%$ en las listas de candidatos de los partidos políticos para la elección de los cuerpos deliberantes. Esta cuota se aplica sólo para los cargos de lista, que son en el caso de diputados aproximadamente la mitad del total, lo que implica que la cuota femenina es del quince por ciento del total de candidatos de los partidos. Tampoco se estableció claramente, aunque los tribunales podrían considerarlo implícito, que esta participación deba reflejarse también en el orden, de modo que de cada tres puestos al menos uno deba ser femenino como sí existe en Argentina (Jones, 1998). En todo caso era de esperar que esta disposición legal estimulara el aumento de la presencia femenina entre los parlamentarios. Más adelante veremos los resultados.

Para la elección del presidente, al igual que los gobernadores de estado, se siguió utilizando el sistema de mayoría relativa en una sola vuelta electoral. El Senado mantuvo su tradicional sistema de listas cerradas y bloqueadas en circunscripciones binominales, con representación proporcional mediante la fórmula de D’Hondt y la adjudicación de cargos adicionales compensatorios (Molina, 1991: 39, 77). De modo que la reforma no extendió al Senado el voto personalizado que se aplica para los diputados al Congreso y las asambleas legislativas. Se eligieron 48 senadores en 24 circunscripciones (23 estados y el Distrito Federal), y resultaron 6 senadores adicionales, para un total de 54. A ellos habrá que sumarles tres senadores vitalicios"

Para la elección de los diputados al Congreso y a las asambleas legislativas de los estados se mantuvo el sistema de representación proporcional personalizada introducido en la ley a partir de 1989, con algunas modificaciones. Se eligieron 189 diputados en 24 circunscripciones (23 estados y el distrito federal), más los escaños adicionales compensatorios. Aproximadamente la mitad de los escaños se elige mediante listas cerradas y bloqueadas, y la otra mitad mediante voto nominal por mayoría relativa, en circunscripciones que pueden ser de un escaño (uninominales) o de más de un escaño (plurinominales). Antes todas eran necesariamente uninominales. Para determinar el número de cargos a elegir en forma nominal se divide el total de cargos de la entidad federal entre dos, y si el resultado no es exacto se redondea

8. Agradecemos el acceso a la base de datos del Latinobarómetro 95 a los profesores Federico Welsch y José Vicente Carrasquero de la Universidad Simón Bolívar, Caracas, Venezuela. La encuesta Latinobarómetro 95 fue administrada en Venezuela por la empresa DOXA, a 1200 personas durante el año 1995 .

9. Se trata de la encuesta Pre-electoral realizada por la Encuestadora Datos para la Red Interuniverstaria de Cultura Política, administrada a una muestra de 1500 personas la última quincena de noviembre de 1998. La pregunta fue: "Hablemos en general de las elecciones en Venezuela. ¿Usted piensa que las elecciones son totalmente limpias, mas o menos limpias, bastante fraudulentas o totalmente fraudulentas?".

10. El cociente nacional electoral para cada cámara se obtiene dividiendo el total de votos válidos emitidos nacionalmente entre el total de cargos asignado al conjunto de las entidades federales.

11. En Venezuela, los ex-presidentes, que no hubieran sido condenados por delitos cometidos en el ejercicio de su cargo, pasan a la condición de senadores vitalicios. Actualmente son: Rafael Caldera, Jaime Lusinchi y Luis Herrera Campíns. 
hacia el entero inferior, antes se redondeaba hacia el superior. El nuevo procedimiento reduce levemente los cargos nominales electos por mayoría en favor de los de lista, electos proporcionalmente. Para la elección de 1998 de los 189 diputados a elegir en las entidades federales, 88 fueron electos por votación personalizada y 101 mediante listas. Para elegir a los 88 diputados en forma personalizada se crearon 72 circunscripciones, 66 unipersonales y 6 plurinominales. De estas últimas, una eligió dos cargos, tres eligieron tres cargos, una eligió cuatro cargos y una eligió siete cargos.

Para cada entidad electoral las organizaciones políticas pueden postular una lista de candidatos y además candidatos para los cargos personalizados. El elector al votar selecciona una lista y tantos candidatos nominales como corresponda a su circunscripción (uninominal o plurinominal). Puede seleccionar la lista de un partido y un candidato de otro. Si se eligen varios cargos nominales puede seleccionar candidatos de una o varias organizaciones.

Para la adjudicación, el procedimiento previsto es el siguiente: se determinan en primer lugar los ganadores de las circunscripciones nominales, y las organizaciones o alianzas a las que pertenecen. A continuación, con base en la votación de cada lista (o alianza de listas) se distribuyen proporcionalmente, utilizando la fórmula de D’Hondt, un número de escaños igual al total (nominales + lista) que corresponden a la entidad. Al número que corresponda a cada lista se le restan los escaños nominales que obtuvo. El resultado es el número de escaños de lista que corresponden a cada organización y se distribuyen en el orden de postulación (listas cerradas y bloqueadas). Si una o varias listas logran más cargos nominales del total que le corresponden proporcionalmente, se queda con estos cargos pero se eliminan los candidatos a los que hubieran correspondido los últimos cocientes en la distribución proporcional. Hasta ahora estos cargos se mantenían, con lo que aumentaba el número total de diputados. Este cambio eliminó en perjuicio de las minorías los llamados por Nohlen (1994: 202,215) "escaños de exceso", ya que los partidos que usualmente logran más cargos nominales de los que proporcionalmente les corresponden son los mayoritarios.

$\mathrm{Al}$ igual que en el senado se adjudicaron diputados adicionales, en un número de 18 , lo que elevó el total de diputados para el próximo Congreso a 207.

\section{LOS PARTIDOS POLÍTICOS}

Los principales partidos tradicionales venezolanos, Acción Democrática y COPEI, enfrentaron en las contiendas electorales de 1998 a nuevos partidos surgidos en función de liderazgos personales y con miras electorales. Así surgen el Movimiento V República (MVR), para apoyar la candidatura presidencial de Hugo Chávez Frías, junto a su principal aliado Patria Para Todos (PPT) una escisión de La Causa R; el Movimiento Irene, de menor trascendencia política, creado para impulsar a la Alcaldesa Irene Sáez, y por último, la conversión en Proyecto Venezuela, para respaldar las candidatura presidencial de Henrique Salas Römer, del llamado Proyecto Carabobo, que había servido de platafor- ma política regional a su hijo, cuando fue elegido gobernador de ese estado en 1995.

La proliferación de estos partidos creados con el objetivo de servir de plataforma a un líder forma parte de un proceso de reversión en la institucionalización del sistema de partidos, utilizando la terminología de Mainwaring y Scully (1995). Tres características que definen un sistema institucionalizado de partidos, antes claramente presentes en el país, como son la estabilidad de la votación, la centralidad de los partidos en los procesos electorales y su amplia penetración social se han visto muy reducidas.

Los antes poderosos partidos tradicionales $\mathrm{AD}$ y COPEI de tendencias socialdemócrata y demócrata cristiana respectivamente, ambos transformados en partidos pragmáticos, se habían convertido en el centro de la crítica por parte de la opinión pública. Tanto sus líderes, como las propias organizaciones enfrentaban un fuerte desprestigio. En estas condiciones acudieron a las contiendas sin el apoyo popular que los había caracterizado y sin la lealtad a toda prueba de sus maquinarias electorales. COPEI optó por apoyar la candidatura de Irene Sáez, con base en su gran popularidad reflejada en las encuestas al inicio de la campaña electoral. Ampliamente conocida como reina de belleza, también había mostrado una imagen de eficiencia en su gestión como alcaldesa de un importante municipio de Caracas, esforzándose por mantenerse independiente de compromisos partidistas. Por primera vez en la historia electoral venezolana una mujer pareció tener posibilidades reales de alcanzar la Presidencia de la República. Por su parte, Acción Democrática lanzó como candidato a uno de sus veteranos Luis Alfaro Ucero, a pesar de su poca notoriedad, y con la imagen de "hombre de aparato". Alfaro perteneciente a la generación de los fundadores del partido, había organizado la eficiente maquinaria electoral de $\mathrm{AD}$, y su autoridad interna parecía indiscutible.

Tanto AD como COPEI confiaban en que las elecciones regionales generarían la adhesión de importantes sectores del electorado, bajo la guía de los líderes partidistas locales y particularmente de los gobernadores, en su gran mayoría pertenecientes a uno de estos dos partidos. Esto consolidaría su posición en los estados y arrastraría la votación hacia sus candidatos presidenciales.

El Movimiento Al Socialismo (MAS), partido de izquierda moderada que había logrado sobrevivir con éxito al largo esquema bipartidista, apoyó a Hugo Chávez en una decisión cuestionada internamente al inicio, pero que logró aceptación a medida que se desarrollaba la campaña electoral. Por su parte, el partido fundado por el Presidente Rafael Caldera en 1993, Convergencia Nacional, muy debilitado durante los cinco años de gobierno, no presentó candidato presidencial propio.

Los partidos que apoyaban la candidatura de Hugo Chávez conformaron el denominado Polo Patriótico, constituido por el MVR, PPT y MAS, junto a un grupo de pequeños partidos (ver Cuadro V). Esta alianza es un conglomerado de tendencias ideológicas que incluye desde la izquierda más tradicional hasta posiciones conservadoras. Su eje, el MVR, es un partido de corte militarista, cuyos dirigentes principales son los oficiales comprometidos con los 
intentos de golpe de estado ocurridos en 1992. Aunque de una gran fortaleza para las elecciones presidenciales, la alianza no funcionó de idéntica manera en las regionales. En algunos casos, el MAS presentó candidaturas diferentes, o apoyó candidatos de otros partidos. Para contrarrestar la fuerza de la candidatura de Chávez se intentó la conformación de un Polo Democrático alrededor de una candidatura única. El logro de este objetivo se vio obstaculizado por la falta de consenso interno y porque Salas no deseaba ser visto como el candidato de las direcciones de AD y COPEI, sino de sus bases y líderes regionales (gobernadores). Finalmente, el acuerdo se logró en la última semana. AD y COPEI abandonaron a sus candidatos para apoyar a Salas. Sin embargo fue un proceso traumático, que no contó con la aprobación ni de Luis Alfaro ni de Irene Sáez, quienes mantuvieron sus candidaturas, con apoyo de partidos menores.

Las condiciones más visibles del sistema de partidos de cara a los comicios regionales y presidenciales eran el desmoronamiento de los partidos tradicionales y la inestabilidad de las nuevas opciones.

\section{DE NUEVO ABSTENCIÓN. DE NUEVO ABSTEN- CIÓN. DE NUEVO ABSTENCIÓN. DE NUEVO ABSTENCIÓN. DE NUEVO ABSTENCIÓN. DE NUEVO ABSTENCIÓN}

La abstención se ha convertido en una característica permanente de las elecciones en Venezuela. A pesar de que hasta 1988 la participación en los comicios nacionales nunca bajó del $80 \%$, en los procesos locales y regionales la abstención presentaba consistentemente altos promedios, como se aprecia en el Gráfico I.

Gráfico I

Abstención en elecciones nacionales y regionales Venezuela 1957-1998
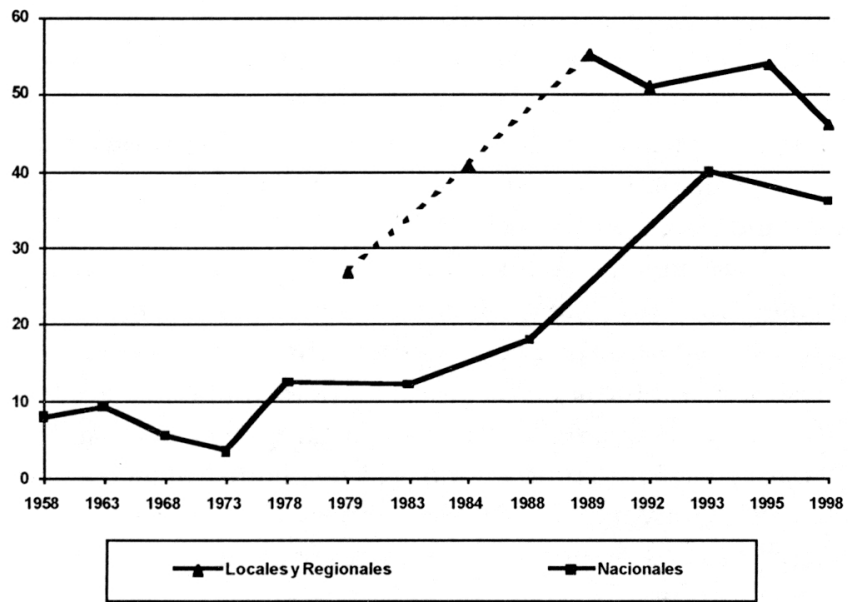

En 1998 la abstención en las elecciones regionales de noviembre se situó en el $46.1 \%$ de los electores registrados ${ }^{12}$, lo cual representa una considerable disminución de ocho puntos porcentuales con respecto a elecciones de la misma naturaleza celebradas en 1995. La cercanía de las presidenciales hizo más atractivas estas elecciones, toda vez que se presentaban como una medición de las fuerzas políticas de los candidatos a la Presidencia. En las elecciones nacionales la abstención se ubicó en el 36.2\%, disminuyendo también aunque en menor proporción (menos de cuatro puntos porcentuales), en relación con las elecciones nacionales de 1993.

Este moderado descenso de la abstención parece responder a la idea de que se trataba de elecciones de importancia vital para el futuro político venezolano. Mas que la elección del próximo mandatario, el ambiente electoral creaba la impresión de enfrentar una decisión sobre profundos cambios y transformaciones que el país necesita, y el estilo y dirigentes con los cuales tales cambios debían ser llevados a cabo. En fin, se enfrentaba una decisión sobre el propio sistema político y la pervivencia de la democracia.

Sin embargo, los factores que desde el inicio de la década de los noventa han determinado altos niveles de abstención, se mantuvieron presentes: una mermada capacidad de los partidos de movilizar a los ciudadanos, y una actitud predominantemente negativa hacia el sistema político y sus principales instituciones. En esta ocasión, el descontento y el resentimiento contra los partidos tradicionales tenían una forma de expresión: el voto por Chávez. Sin embargo, es probable que el estímulo hacia la participación propiciado por este factor, haya sido neutralizado por la continua erosión de la capacidad de movilización de los partidos, ya sea por el desprestigio de los tradicionales, o por la ausencia de lealtades sólidas hacia los nuevos. Este balance ayuda a mantener los porcentajes de abstención en niveles similares con respecto a 1993.

\section{LAS ELECCIONES DE GOBERNADORES Y DIPUTADOS A LAS ASAMBLEAS LEGISLATIVAS Y AL CONGRESO NACIONAL DEL 8 DE NOVIEMBRE DE 1998}

\section{La campaña electoral}

A pesar de que se trataba de dos procesos electorales diferentes, la campaña electoral de uno y otro se entremezclaron. En el nivel regional, las campañas estuvieron centradas en la evaluación de la gestión de los gobernadores en cargo, puesto que casi todos optaban por la reelección. Si bien los temas locales fueron los más importantes en cada estado, el apoyo a las opciones regionales parecía implicar un respaldo a sus aliados, partidos o movimientos, en el nivel nacional. Lo que sí aparecía como obvio era que de los resultados electorales regionales, dependía la estrategia de los partidos y candidatos ante las elecciones presidenciales. De hecho, pasadas las elecciones de noviembre se redefinieron las candidaturas presidenciales.

La cercanía de ambos procesos tuvo influencias mutuas. Por una parte, la intención de los partidos tradicionales al separar la elección estuvo en pretender que el voto por los gobernadores tuviera un efecto de arrastre sobre el voto para diputados y senadores al Congreso Nacional, y lógicamente para los miembros de las asambleas legislativas, lo cual en

12. La abstención en relación con la población electoral en general (inscrita o no en el Registro Electoral Permanente) es aproximadamente un seis por ciento más alta. 
efecto se logró. Sin embargo, también se pretendía con esta separación de los comicios el establecimiento de una imagen de fortaleza para AD y COPEI que creara un clima político favorable a los candidatos presidenciales que apoyaban estos partidos. En este sentido, esta estrategia no rindió los frutos esperados, quedando demostrada la declinación del voto comprometido con los partidos, propio de lealtades partidistas firmes que en Venezuela han perdido fuerza. Por otra parte, los partidos realizaron las más variadas alianzas para apoyar a los gobernadores, en función de situaciones políticas específicas de cada estado, lo cual complicaba aún más el panorama electoral.

\section{Análisis de los resultados}

El 8 de noviembre de 1998 se produjeron las elecciones de los gobernadores de 23 estados, los diputados a las asambleas legislativas de los estados, y los diputados y senadores al Congreso Nacional, a través de un instrumento de votación sumamente confuso y difícil de utilizar. Esto, la utilización por primera vez de máquinas escrutadoras para automatizar el proceso y la conformación de las mesas electorales por ciudadanos elegidos al azar (no representantes de los partidos), a quienes no se notificó a tiempo ni se entrenó adecuadamente, retardó la instalación de las mesas, haciendo en muchos casos el proceso lento, complicado y engorroso para los electores. El cuadro II muestra los resultados de las elecciones a gobernador comparados con los resultados de 1995, tanto en porcentaje de votos de los partidos, como en número de gobernadores electos.

\section{Cuadro II}

ELECCIONES DE GOBERNADOR DE 1995 Y 1998 RESULTADOS POR PARTIDO

\begin{tabular}{lrccc}
\hline \multicolumn{1}{c}{ Partido } & \multicolumn{2}{c}{1995} & \multicolumn{2}{c}{$\mathbf{1 9 9 8}^{(\mathrm{a})}$} \\
\hline & $\%$ de & Cargos & $\%$ de & Cargos \\
votos $^{(\mathrm{b})}$ & logrados & votos $^{(\mathrm{b})}$ & $\begin{array}{c}\text { logrados } \\
\text { AD }\end{array}$ \\
COPEI & 33.9 & 11 & 28.2 & 8 \\
MAS & 22.5 & 4 & 15.1 & 5 \\
Causa R & 10.7 & 4 & 10.4 & 3 \\
Convergencia & 11.1 & 1 & 3.7 & 1 \\
$\begin{array}{l}\text { Proyecto Carabobo/ } \\
\text { Proyecto Venezuela }\end{array}$ & 8.9 & 1 & 2.5 & 1 \\
PPT & 2.1 & 1 & 6.0 & 1 \\
MVR & & & & \\
Otros & & & 3.1 & 3 \\
\hline
\end{tabular}

(a) En 1998 se incluye al recién creado Estado Vargas.

(b) El porcentaje de votos por partido está calculado sobre el total de votos válidos por gobernadores de todo el país.

(c) Proyecto Carabobo participó en las elecciones regionales de 1995 en ese único estado. Se incluye porque constituye el embrión de Proyecto Venezuela.

Fuentes: CSE, Dirección General de Estadísticas Electorales. Resultados de Gobernadores, 1996 CNE, Resultados Electorales. Venezuela 1998. CD Rom

Tal como lo esperaban al propulsar la separación de los comicios, AD y COPEI parecían haber salido favorecidos en el nivel regional: más de la mitad de las gobernaciones fueron obtenidas por candidatos de estos dos partidos. Sin embargo, la notable reducción de su votación general entre 1995 y 1998, y el hecho de que AD perdiera el control de cuatro gobernaciones (ganando apenas una adicional) pone en duda el aparente éxito de estos partidos. Los otros partidos que habían logrado elegir a sus candidatos en 1995 (MAS, Convergencia, Causa R y Proyecto Venezuela) mantienen sus posiciones. El Movimiento V República alcanza sólo un gobernador propio, pero fue factor decisivo en el éxito de los tres del PPT, el de Causa R 13, y uno de los del MAS.

Como producto del proceso de descentralización política, desde 1989 la elección directa de los gobernadores ha modificado profundamente el panorama político en Venezuela, conformándose los estados en espacios políticos relativamente autónomos de la política nacional. Asimismo, se han establecido nuevas bases para la decisión electoral, relacionadas más con la evaluación de las gestiones de los gobernadores, su imagen y su poder de convencimiento a electorados más reducidos y más puntuales. Estas elecciones de noviembre de 1998 reafirman esta tendencia, permitiendo a partidos políticos como el MAS, Convergencia o Causa R mantener su predominio en algunos estados, a pesar su menor éxito en el nivel nacional.

La reelección fue otro elemento importante en estos resultados. Los gobernadores pueden ser reelectos por un período, y en 1998 de los veinte mandatarios regionales que se presentaron para un segundo mandato de tres años, diecisiete lo lograron, lo cual es indicativo de que sus gestiones fueron valoradas como exitosas, y de la influencia que han obtenido en el nivel regional.

En relación con las Asambleas Legislativas de los estados, es claro que el voto a gobernador tuvo un efecto de arrastre sobre ellas, tal como se aprecia en los resultados, donde las variaciones entre ambas votaciones son pequeñas.

Cuadro III

VOTO POR PARTIDOS EN ELECCIONES DE GOBERNADOR, ASAMBLEAS LEGISLATIVAS Y CONGRESO NACIONAL 8 DE NOVIEMBRE DE 1998

\begin{tabular}{lccc}
\hline \multicolumn{1}{c}{ Partido } & $\begin{array}{c}\text { \% de votos } \\
\text { Gobernador }\end{array}$ & $\begin{array}{c}\text { \% de vostos } \\
\text { Asambleas Legislativas }\end{array}$ & $\begin{array}{c}\text { \% de vostos } \\
\text { Congreso Nacional }\end{array}$ \\
\hline AD & 28.3 & 26.3 & 24.1 \\
MVR & 14.3 & 15.4 & 19.9 \\
COPEI & 15.1 & 14.1 & 12.0 \\
Proyecto Venezuela & 6.0 & 7.7 & 10.4 \\
MAS & 10.4 & 9.9 & 8.9 \\
CAUSA R & 3.7 & 2.6 & 3.0 \\
CONVERGENCIA & 2.5 & 2.7 & 2.5 \\
\hline
\end{tabular}

Fuente: Consejo Nacional Electoral, Resultados Electorales. Venezuela. CD Rom.

Los porcentajes de voto para el Congreso Nacional reflejan la influencia de las elecciones de gobernadores, las variaciones no son muy marcadas entre las tres elecciones, lo cual sugiere que la mayoría de los electores tomó una decisión única para los tres votaciones. Sin embargo, las diferencias que se aprecian revelan, a su vez, el efecto de la cercanía de las presidenciales. Los partidos tradicionales (AD, COPEI y MAS) disminuyen su votación para el Congreso con respecto a las gobernaciones y asambleas legislativas, mientras que los partidos emergentes, Proyecto Venezuela y MVR, la aumentan ligeramente. Los candidatos presidenciales de estas dos últimas organizaciones motivaron el apoyo hacia

13. El gobernador atribuido a Causa R, Francisco Arias Cárdenas es un independiente vinculado a esta organización desde su primera elección. Fue el segundo jefe del intento de golpe militar del 4 de febrero de 1992, es un aliado muy cercano del Presidente Chávez. 
sus candidatos al Congreso, como expresión de respaldo a sus opciones y como una forma dé contribuir a darle fuerza parlamentaria a sus eventuales gestiones.

Es en los resultados de las elecciones a diputados y senadores al Congreso Nacional donde se evidencia más claramente la conformación de un sistema multipartidista inestable (Cuadro IV). En primer lugar, se aprecia un mayor fraccionamiento del sistema de partidos. El estadístico que mide el grado de fraccionamiento es el "Número efectivo de partidos" ${ }^{14}$ ("N"), el cual aumenta de 3.4 en 1988 a 5.6 en 1993, para situarse en 7.5 en esta elección. Esto es indicativo de que un mayor número de partidos comparte las preferencias de los electores. El sistema bipartidista que dominó durante los años setenta y ochenta pierde vigencia para dar paso a uno multipartidista.

Cuadro IV

RESULTADOS DE LAS ELECCIONES AL

CONGRESO NACIONAL DE 1993 Y 1998

VOTO POR PARTIDOS ${ }^{(a)}$

\begin{tabular}{|c|c|c|c|c|c|c|c|c|}
\hline \multirow{3}{*}{ PARTIDO } & \multicolumn{4}{|c|}{ DIPUTADOS } & \multicolumn{4}{|c|}{ SENADORES } \\
\hline & \multicolumn{2}{|c|}{1993} & \multicolumn{2}{|c|}{1998} & \multicolumn{2}{|c|}{1993} & \multicolumn{2}{|c|}{1998} \\
\hline & $\begin{array}{c}\% \\
\text { Votos }\end{array}$ & $\begin{array}{c}\text { No. } \\
\text { Cargos }\end{array}$ & \begin{tabular}{|c|}
$\%$ \\
Votos \\
\end{tabular} & \begin{tabular}{|c|} 
No. \\
Cargos
\end{tabular} & $\begin{array}{c}\% \\
\text { Votos }\end{array}$ & $\begin{array}{c}\text { No. } \\
\text { Cargos }\end{array}$ & \begin{tabular}{|c|}
$\%$ \\
Votos
\end{tabular} & $\begin{array}{l}\text { No. }{ }^{(b)} \\
\text { Cargos }\end{array}$ \\
\hline $\mathrm{AD}$ & 23.3 & 55 & 24.1 & 61 & 24.1 & 16 & 24.4 & $21(1)$ \\
\hline MVR & & & 19.9 & 35 & & & 19.7 & 8 \\
\hline COPEI & 22.6 & 53 & 12.0 & 26 & 22.8 & 14 & 12.1 & $6(1)$ \\
\hline Proyecto Venezuela & & & 10.4 & 20 & & & 10.2 & 3 \\
\hline MAS & 10.8 & 24 & 8.9 & 24 & 10.9 & 5 & 9.1 & 5 \\
\hline PPT & & & 3.4 & 11 & & & 3.4 & 4 \\
\hline \begin{tabular}{|l} 
Causa R \\
\end{tabular} & 20.7 & 40 & 3.0 & 5 & 20.8 & 9 & 3.0 & 1 \\
\hline Convergencia & 13.6 & 26 & 2.5 & 6 & 13.4 & 6 & 2.3 & $3(1)$ \\
\hline Apertura & & & 1.5 & 3 & & & 2.4 & 1 \\
\hline Renovación & & & 1.2 & 2 & & & 1.2 & 1 \\
\hline IRENE & & & 1.3 & 3 & & & 1.2 & \\
\hline Otros & 9.0 & 5 & 11.8 & 11 & 8.0 & 0 & 11.3 & 1 \\
\hline Total & & 203 & & 207 & & 50 & & 57 \\
\hline Partidos con cargos & & 10 & 2 & 21 & & 5 & & 11 \\
\hline Volatilidad & & $2 \%$ & & $1 \%$ & & & & \\
\hline No. efectivo de partidos & & 5.6 & & .6 & & & & \\
\hline
\end{tabular}

(a) Debido a las alianzas, es corriente que se clasifique algunos parlamentarios en partidos que no son los suyos. El Consejo Nacional Electoral tiende a clasificarlos en el partido de mayor votación en la alianza. A fin de determinar el número correcto de cargos por partido se utilizó la autoidentificación partidista de los parlamentarios en una encuesta realizada a cada uno de ellos por El Universal, y facilitada por el periodista Carlos Subero.

(b) Entre paréntesis los senadores vitalicios.

Fuentes: CSE, CNE. Resultados Electorales 1998. CD ROM.

Esta nueva conformación no parece ser muy estable, dado que la votación de los partidos no se mantiene de una elección a otra en forma consistente. Apenas dos partidos conservan su votación entre 1993 y 1998: AD y el MAS. Dos organizaciones que no compitieron en 1993 ingresan con fuerza como nuevos partidos de significación, el MVR y Proyecto Venezuela. Finalmente tres de los partidos importantes en el periodo pasado se descalabran electoralmente: COPEI, Causa $\mathrm{R}$ y Convergencia, que ven disminuir su votación en más de diez puntos de porcentaje. En 1988 la volatilidad fue de $8 \%$, en 1993 de $32 \%$ y en 1998 llega al 41\%. Los partidos que en 1993 lograron resquebrajar el sistema bipartidista han sido rápidamente desplazados por nuevos movimientos de similares características. La volatilidad de las decisiones electorales se ha convertido en un nuevo ingrediente del comportamiento político venezolano.
En relación con la incorporación de las mujeres, el Congreso electo cuenta con 24 diputadas y 5 senadoras, once por ciento del total. En el parlamento anterior la presencia femenina fue del $7 \%$. Ello parece indicar que la cuota establecida en la reforma electoral, a la que hicimos referencia previamente, tuvo alguna eficacia, aún cuando se está lejos de alcanzar siquiera el quince por ciento de presencia al que apunta la misma.

Estas elecciones regionales, a pesar de su singularidad dada la cercanía de las elecciones presidenciales y de incluir en el mismo proceso a los diputados y senadores del Congreso, lograron cierto grado de autonomía con respecto a las nacionales. En lugar de ser totalmente arrastradas por el voto nacional, ayudaron a la conformación del escenario presidencial.

\section{LAS ELECCIONES PRESIDENCIALES DEL 6 DE DICIEMBRE DE 1998}

\section{La Campaña Electoral}

El tema central de la campaña fue el del "cambio". Cambio fundamentalmente respecto al gobierno y la influencia gubernamental de los partidos tradicionales Acción Democrática y COPEI, así como de las instituciones y políticas desarrolladas por ellos. Las dos candidaturas que terminaron polarizando el voto popular presentaron como sus consignas centrales la revolución democrática mediante una Asamblea Constituyente, en el caso de Chávez, y el cambio radical, con la profundización de la descentralización como idea principal, por parte de Henrique Salas Römer. En ese sentido, para muchos electores su decisión de voto giró en torno a saber cual de las dos candidaturas garantizaba mejor la posibilidad de un cambio realmente profundo.

Al lado del tema del cambio, se debatió sobre el carácter democrático del mismo. Los adversarios de Hugo Chávez centraron su ataque en el contenido autoritario y antidemocrático tanto de su mensaje, como de su posible gobierno, así como en las credenciales democráticas de su principal adversario que había previamente sido gobernador de uno de los principales estados del país.

\section{Análisis de los resultados}

El resultado de la elección, una victoria para Hugo Chávez con el $56.2 \%$ de los votos como puede verse en el Cuadro $\mathrm{V}$, indica que su candidatura fue percibida como una opción más adecuada para el cambio, y que no fue convincente la campaña sobre su supuesta condición antidemocrática. A ello ayudaron los errores de sus adversarios. El principal de ellos fue la aceptación por parte de Salas Römer del apoyo de los partidos tradicionales de gobierno, lo que

14. El "Número Efectivo de Partidos (N)" de Laakso y Taagepera (1979) indica cual sería el número de partidos con votación igual al que es equivalente la distribución de la votación entre los partidos ocurrida en una elección en particular. Es el estadístico más utilizado para medir el grado de dispersión o concentración del sistema de partidos (Molina, 1991: 94). En este caso se refiere a la distribución de los votos lista para la Cámara de Diputados. 
le hizo perder credibilidad en su oferta de cambio y movió, sobretodo a los electores independientes, hacia la alternativa que más claramente lo garantizaba. Hasta marzo de 1998 Irene Sáez aparecía adelante en las encuestas de opinión con una ventaja clara sobre Hugo Chávez y Henrique Salas Römer. Cuando aceptó el apoyo de COPEI inmediatamente comenzó a descender hasta quedar sin oportunidad alguna. Sus limitaciones para la comunicación igualmente influyeron, pero ésta que señalamos fue la causa más importante de su caída en picada en los sondeos y en la voluntad popular. Luego de las elecciones parlamentarias y a menos de una semana de las elecciones, Acción Democrática y COPEI ante la polarización en torno a Chávez y Salas, deciden abandonar sus candidatos y apoyar al último de ellos. Esto fue un beso de la muerte. Los sondeos realizados por la empresa Datanalysis para El Universal y presentados por el periodista Carlos Subero en su página de internet (http://207.87.9100/ Flash2/sld001.htm) muestran como a raíz de ese apoyo la candidatura de Salas, que nunca llegó a superar a la de Chávez, sufre una reducción de sus preferencias entre los independientes, que no fue suficientemente compensada por el incremento de la votación entre los partidarios de Acción Democrática y COPEI. La explicación más plausible de ello es que a los ojos de una parte significativa del electorado, $\mathrm{AD}$ y COPEI eran la piedra de toque para determinar si se era partidario de la continuidad o del cambio. La asociación con ellos generó inmediatamente dudas sobre la credibilidad del cambio ofrecido por Salas, dio apoyo a la propaganda del Polo Patriótico que acusaba a Salas de ser "más de lo mismo", y terminó favoreciendo la candidatura de Chávez.

Cuadro V

RESULTADO DE LAS ELECCIONES PRESIDENCIALES DE VENEZUELA VOTACIÓN DE LOS CANDIDATOS 6 DE DICIEMBRE DE 1998

\begin{tabular}{|c|c|c|}
\hline Candidato & $\%$ de Votos & Partidos que lo apoyan y sus porcentajes de votos \\
\hline Hugo Chávez & 56.20 & $\begin{array}{l}\text { MVR }(40.17 \%) \text {; MAS }(9 \%) \text {; PPT }(2.19 \%) \text { PCV } \\
(1,25 \%) \text {, IPCN }(1.03 \%) \text {; GE }(0.86 \%) \text {; MEP }(0.84 \%) \text {; } \\
\text { SI }(0.57 \%) \text {; AA }(0.29 \%) \text {. }\end{array}$ \\
\hline $\begin{array}{l}\text { Henrique Salas } \\
\text { Römer }\end{array}$ & 39.97 & $\begin{array}{l}\text { Proyecto Venezuela }(28.75 \%) \text {; AD }(9.05 \%) \text {; COPEI } \\
(2.15 \%) \text {; PQAC }(0.02 \%) \text {. }\end{array}$ \\
\hline Irene Sáez & 2.82 & $\begin{array}{l}\text { IRENE }(1.96 \%) \text {; FD }(0.37 \%) \text {; La Llave }(0.30 \%) \text {; } \\
\text { INCVF }(0.19 \%) \text {. }\end{array}$ \\
\hline Luis Alfaro Ucero & $0.42 \%$ & $\begin{array}{l}\text { O.R.A. }(0.12 \%) \text {; U.R.D. }(0.08 \%) \text {; RENACE }(0.08 \%) \text {; } \\
\text { VU }(0.05 \%) \text {; ICC }(0.05 \%) \text {; FIN }(0.03 \%) \text {; ONDA } \\
(0.01 \%)\end{array}$ \\
\hline Miguel Rodríguez & $0.30 \%$ & APERTURA $(0.30 \%)$ \\
\hline Alfredo Ramos & $0.11 \%$ & La Causa R $(0.11 \%)$ \\
\hline Otros & $0.18 \%$ & \\
\hline \multicolumn{2}{|c|}{ Total Votantes: $6.988 .291(63.76 \%)$} & Votos Válidos: $93.55 \%$ \\
\hline \multicolumn{2}{|c|}{ Abstención: $3.971 .239(36.24 \%)$} & Votos Nulos: $6.45 \%$ \\
\hline
\end{tabular}

Nota: El nombre completo de los partidos aparece en el Anexo I: Partidos Políticos Venezolanos

Fuente: Consejo Nacional Electoral (Con 99.61\% de los votos escrutados, el 10 de diciembre de 1998.). Página Web: http://www.elecciones98.cantv.net.

Un factor importante para la elección presidencial fue sin duda el resultado de la elección parlamentaria separada. Este resultado ratificó a Chávez y a la alianza que lo respaldaba como la principal opción para las elecciones presidenciales. Al mismo tiempo complicó la situación de Salas, ya que hizo dudar de que la polarización que señalaban las encuestas entre su candidatura y la de Chávez fuera real.
Acción Democrática intentó utilizar su primer lugar en la votación parlamentaria como palanca para impulsar la candidatura de Alfaro, y COPEI en un primer momento pareció inclinarse a apoyarlo. Ello, a nuestro modo de ver también ayudó a Chávez, ya que para muchos electores aunque la primera opción era Salas, si la alternativa era entre Alfaro o Chávez, preferían al segundo para buscar el cambio. Como se dijo, al final $A D$ y COPEI reconocieron la polarización, respaldaron a Salas y retiraron el apoyo a sus candidatos.

Los cuadros $\mathrm{V}$ y VI presentan el resultado de la elección presidencial, el primero indica la votación de los candidatos y los partidos que los apoyaron. El segundo compara los sufragios obtenidos por los partidos en 1998 con lo sucedido en 1993. Este resultado es un reflejo del profundo descontento de la población con la situación social y económica del país tal coma la presentamos en los antecedentes, pero además de las modificaciones que se han producido en los patrones de comportamiento electoral y a los cuales también hicimos referencia antes. Como puede observarse, no sólo la elección se polarizó entre dos candidaturas que no provenían de los partidos tradicionales, sino que en la votación por partido el $70 \%$ de los votos es acumulado por organizaciones que giran en torno al liderazgo de estos candidatos, organizaciones que aún no tienen peso ni respaldo propio más allá del de sus líderes, y que podrían perder significación si se opaca la estrella de éstos, tal como ocurrió con La Causa R y Convergencia. Así pues la elección acentúa la personalización de la política.

Cuadro VI

ELECCIONES PRESIDENCIALES VENEZOLANAS DE 1993-1998 COMPARACIÓN DE LOS RESULTADOS POR PARTIDO

\begin{tabular}{|c|c|c|}
\hline \multirow[t]{2}{*}{ PARTIDO } & \multicolumn{2}{|c|}{ PORCENTAJE DE VOTOS $^{(\mathrm{a})}$} \\
\hline & 1993 & 1998 \\
\hline Movimiento V República & -.---- & $40.17 \%$ \\
\hline Proyecto Venezuela & ------ & $28.75 \%$ \\
\hline Acción Democrática & $23.23 \%$ & $9.05 \%$ \\
\hline Movimiento Al Socialismo & $10.59 \%$ & $9.00 \%$ \\
\hline Patria Para Todos & $21.95 \%{ }^{(\mathrm{b})}$ & $2.19 \%$ \\
\hline La Causa Radical & & $0.11 \%$ \\
\hline COPEI & $22.11 \%$ & $2.15 \%$ \\
\hline IRENE & -..-..-- & $1.96 \%$ \\
\hline Convergencia & $17.03 \%$ & 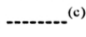 \\
\hline PCV & $0.34 \%$ & $1.25 \%$ \\
\hline IPCN & -.--- & $1.03 \%$ \\
\hline Otros & $4.75 \%$ & $4.34 \%$ \\
\hline Volatilidad & $48 \%$ & $75 \%$ \\
\hline Número Efectivo de Partidos & 5.2 & 3.8 \\
\hline
\end{tabular}

(a) Se incluyen los partidos que obtuvieron el $1 \%$ o más de los votos válidos en alguna de las dos elecciones.

(b) Patria Para Todos se produce mediante una división de La Causa Radical con posterioridad a las elecciones de 1993. Por ello la votación de esta elección corresponde a ambos grupos.

(c) Convergencia no postuló candidato para las elecciones presidenciales, aunque le dieron su apoyo a Henrique Salas Römer, extraoficialmente.

La volatilidad igualmente se acentúa. El Cuadro VI nos muestra como el porcentaje de votos que cambia de unos a otros partidos es para 1998 de $75 \%$, aún mayor que el ya alto del $48 \%$ observado en 1993 , ambos muy superiores a la situación de estabilidad característica de la época del bipartidismo. La volatilidad para la elección presidencial de 1988 fue de $7 \%$, similar a las anteriores. Un elemento cambia y es el relativo a la concentración del voto. En las elecciones de 
1993 el voto se distribuye en cinco partidos tanto en las parlamentarias como en las presidenciales. Entonces sostuvimos que el sistema electoral de mayoría relativa utilizado para las elecciones presidenciales estimularía la concentración del voto y la reducción del número de partidos relevantes electoralmente. La elección parlamentaria separada de la presidencial confirmó el multipardismo, y presentó como vimos un Número Efectivo de Partidos de 7.6, aún más alto que el de 1993. En la presidencial comienza a observarse el efecto de concentración que estimula, entre otros factores el sistema electoral, la votación se concentra básicamente en dos partidos con votación alta, y dos con votación mediana, para un Número Efectivo de Partidos de 3.8, menor al ocurrido en 1993 (5.2). La volatilidad electoral y la incertidumbre con relación a si se mantendrá el mismo sistema electoral hacen difícil predecir si se continuará por el camino de la concentración del voto y una posible reconstitución de un bipartidismo estable. Hasta que no se consoliden las nuevas opciones parece más probable, si se mantiene el sistema electoral de mayoría relativa en el nivel presidencial y se vuelve a las elecciones parlamentarias simultáneas, que estemos ingresando a una situación de bipartidismo inestable, en la cual en cada elección dos partidos aglutinan mayoritariamente al electorado, pero los protagonistas cambian de una elección a otra.

\section{CONCLUSIONES}

Las elecciones de 1998 tuvieron para Venezuela la dramática significación que puede atribuirse a una encrucijada histórica. Y aunque sea posible referir a 1993 el comienzo de la ruptura con el modelo de dominación política originado en el acuerdo de Punto Fijo, lo cierto es que ahora la opción fue mucho más clara y la decisión contundente como un hachazo. El deterioro de la situación económica y social a partir de los años ochenta, la incapacidad de los partidos tradicionales para darle una respuesta satisfactoria, según el juicio recogido en múltiples sondeos, los sucesivos escándalos en torno a la corrupción de altos funcionarios públi$\cos$, la crisis política y de legitimidad que emerge en 1992, el fracaso del gobierno de Caldera en responder satisfactoriamente a las expectativas de transformación que lo llevaron al poder, fueron los afluentes que condujeron a este cruce de caminos. Las alternativas podían resumirse en tres: la continuidad, representada por Luis Alfaro Ucero (AD) e Irene Sáez (COPEI); el cambio moderado, postulado por Henrique Salas Römer (Proyecto Venezuela) y el cambio profundo, encarnado por Hugo Chávez (Movimiento V República, Movimiento Al Socialismo, Patria Para Todos y otros).

La victoria de Hugo Chávez ciertamente implica una ruptura, aunque no dejan de haber signos de continuidad. Es en primer lugar una ruptura en el sentido de que el liderazgo cuyo origen puede referirse al pacto fundador de la democracia en 1958 es sustituido por una alianza entre la izquierda excluida entonces y los militares rebeldes de 1992. Se trata claramente de un nuevo pacto político, aún en proceso de consolidación. Cambia también la relación del núcleo gobernante con los factores sociales. Un apoyo fundamen- tal del Pacto de Punto Fijo estaba constituido por la primera central obrera del país, la Confederación de Trabajadores de Venezuela, controlada por Acción Democrática, ahora ésta ha sido expresamente excluida del diálogo por el nuevo gobierno, que ha intentado una vinculación directa con el "pueblo soberano" y aspira a reconstituir el movimiento sindical. Es también una ruptura por la función que se le da a las Fuerzas Armadas, las cuales adquieren un papel mucho más directo en el ejercicio del poder, y de custodios del "buen gobierno", ante la exaltación oficial de los intentos de golpe de estado del 4 de febrero y el 27 de noviembre de 1992. Los empresarios, aparentemente, mantienen su vinculación privilegiada y directa como copartícipes de la política económica y social ${ }^{15}$. También puede hablarse de continuidad en la deferencia hacia la Iglesia Católica, y su aceptación como un importante interlocutor.

Un aspecto a considerar es el relativo a si la ruptura institucional, marcada por la apertura de un proceso constituyente, la apelación directa al pueblo por encima de los partidos políticos y la intensificación de la presencia política de las Fuerzas Armadas, implica una desconsolidación y eventual sustitución del sistema democrático. Al menos desde el punto de vista de la población este no parece ser el caso. El apoyo al presidente Chávez siempre ha sido acompañado en los sondeos de opinión por un alto respaldo a la democracia. La encuesta nacional realizada entre la elección parlamentaria y la presidencial por la Red Interuniversitaria de Cultura Política ${ }^{16}$, en noviembre de 1998, confirma esta tesis. Ante la pregunta: "Y que prefiere usted, ¿una democracia como la que tenemos o una dictadura?". Las respuestas fueron: democracia $78.2 \%$; depende $10.8 \%$; dictadura $7.2 \%$; ninguna de las dos $2.6 \%$; no sabe o no contesta $1.2 \%$. Asimismo, ante la pregunta: "Piensa usted que a pesar de los problemas que tiene la democracia venezolana, debe mantenerse o debe ser sustituida?", los entrevistados respondieron: deber ser sustituida $35.5 \%$; debe mantenerse $59.7 \%$, no sabe o no contesta 2.8. Más aún del $35.5 \%$ que afirma debe ser sustituida el $85 \%$ quiere verla sustituida con una democracia distinta ("fuerte" o "social") y no con una dictadura. Es decir, que a juzgar por estos datos la opinión pública será un factor favorable a la democracia dentro del proceso de transformación institucional. En otras palabras, la elección del 6 de diciembre no puede interpretarse como un mandato para el desmantelamiento de la democracia, aunque sí para el cambio. Sin embargo, es obvio que una opinión pública inclinada democráticamente no es suficiente

15. Un signo de ello es que ante la exclusión de la principal central obrera en las conversaciones sobre salario mínimo, el gobierno lo fijó unilateralmente para el sector público, pero decidió negociar con los empresarios el relativo al sector privado. El Ministerio de Agricultura y Cría fue asignado a un empresario agrícola, y en el Ministerio de Hacienda se mantuvo a la funcionaria del gobierno anterior, con vinculaciones directas con los organismos internacionales y del agrado de los industriales.

16. La Red Interuniversitaria de Cultura Política es un Grupo de Investigación académica financiado por el CONICIT, en el cual participan especialistas de la Universidad Central de Venezuela, la Universidad Simón Bolivar, el Instituto de Estudios Superiores de Administración y la Universidad del Zulia. La encuesta fue administrada por la empresa Datos a una muestra de 1500 personas, durante la segunda quincena de noviembre de 1998, entre la elección de noviembre y la de diciembre. 
para contrarrestar tendencias al autoritarismo y la concentración del poder que pudieran cobijarse bajo un liderazgo carismático y mecanismos plebiscitarios. Dada la heterogeneidad del "Polo Patriótico", y la incertidumbre en torno a la capacidad de la oposición para enfrentarlo con un apoyo popular significativo, es imposible predecir el camino que tomarán los acontecimientos.

En el campo de la economía el gobierno que nunca presentó ideas claras durante su campaña electoral, se inauguró acentuando la continuidad con el programa de la anterior administración. El cambio más notable fue la eliminación del diálogo tripartito (empresarios, sindicatos, gobierno) como mecanismo de fijación de parámetros básicos de la política salarial, aparentemente con la intención de acentuar una relación privilegiada gobierno-empresarios, lo cual le daría un giro a la derecha a la política económica y social, contradictorio no sólo con las promesas de hacer de la lucha contra la pobreza el eje de la acción gubernamental, sino también con las expectativas de un electorado volátil, que podría en cualquier momento dejar abandonado a sus amores de hoy. En conclusión, ante la encrucijada, los electores decidieron tomar el camino del cambio revolucionario en democracia, lo que aún no está claro es si la ruta conduce en la dirección deseada.

\section{ANEXO I \\ PARTIDOS POLITICOS VENEZOLANOS \\ PARTICIPANTES EN LAS ELECCIONES DE 1998}

\begin{tabular}{|c|c|}
\hline AA & Acción Agropecuaria \\
\hline $\mathrm{AD}$ & Acción Democrática \\
\hline APERTURA & Apertura a la Participación Nacional \\
\hline CONVERGENCIA & Convergencia Nacional \\
\hline COPEI & Comité de Organización Política Electoral Independiente \\
\hline DR & Democracia Renovadora \\
\hline FD & Factor Democrático \\
\hline FIN & Fuerza Independiente Nacional \\
\hline FS & Frente Soberano \\
\hline GE & Gente Emergente \\
\hline ICC & Independientes Con el Cambio \\
\hline IPCN & Independientes por la Comunidad Nacional \\
\hline IPV & Independientes por Venezuela \\
\hline IRENE & Integración Representación Nueva Esperanza \\
\hline La Causa R & La Causa Radical \\
\hline La Llave & La Llama de Venezuela \\
\hline MAS & Movimiento Al Socialismo \\
\hline MDP-BR & Movimiento por la Democracia Popular - Bandera Roja \\
\hline MEP & Movimiento Electoral del Pueblo \\
\hline MIN & Movimiento de Integridad Nacional \\
\hline MR & Movimiento Republicano \\
\hline MVR & Movimiento V (Quinta) República \\
\hline ONDA & Organización Nacionalista Democrática Activa \\
\hline OPINA & Opinión Nacional \\
\hline ORA & Organización Renovadora Auténtica \\
\hline PCV & Partido Comunista de Venezuela \\
\hline PPT & Patria Para Todos \\
\hline PQAC & Por Querer a la Ciudad \\
\hline PRVZL & Proyecto Venezuela \\
\hline RENACE & Rescate Nacional Electoral \\
\hline RENOVACIÓN & Renovación \\
\hline SI & Movimiento Solidaridad Independiente \\
\hline SOLUCION & $\begin{array}{l}\text { Sociedad Luchadora con Independientes Organizados } \\
\text { por la Nación }\end{array}$ \\
\hline UDH & Unidos por los Derecho Humanos \\
\hline URD & Unión Republicana Democrática \\
\hline VU & Venezuela Unida \\
\hline
\end{tabular}

\section{BIBLIOGRAFÍA}

Alvarez, Angel (1994): Estrategias de Propaganda Electoral. Caracas: Instituto de Estudios Políticos de la Universidad Central de Venezuela.

BalOYRA, Enrique (1993): "Venezuela. Elecciones generales 5 de diciembre de 1993". Boletín Electoral Latinoamericano $X$, julio-diciembre, pp. 31-42.

Baloyra, E. y J. Martz (1979): Political Attitudes in Venezuela. Austin: University of Texas Press.

BAPTISTA, Asdrubal (1997): "Medidas de la desigualdad en la distribución del ingreso”. En Datos Económicos de Venezuela. Diagnóstico Estadístico Social. Caracas: Fundación Centro Gumilla. Revista SIC. Versión diskette.

Dalton, R. J. y M. P. Wattemberg (1993): "The Not So Simple Act of Voting", en Ada W Finifter (ed.) Political Science: The State of the Discipline II; Washington, DC: American Political Science Association.

HidAlgo, Manuel (1998): "Consolidación, crisis y cambio del sistema venezolano de partidos”. Politeia, 21, Caracas: Instituto de Estudios Políticos de la Universidad Central de Venezuela, pp 63-104.

JONES, Mark (1998): “Gender Quotas, Electoral Laws, and the Election of Women. Lessons From the Argentine Provinces”. Comparative Political Studies 31, 1, febrero, pp. 3-21.

LAAKSO, Markku y Rein TAAgEPERA (1979): "The Effective Number of Parties: A Measure with Application to Western Europe." Comparative Political Studies 12 (1), pp 3-27.

MAINGON, Thais y Tanaly PATRUYO (1996): "Las elecciones locales y regionales de 1995: tendencias políticas". Cuestiones Políticas, 16, Maracaibo: Instituto de Estudios Políticos y Derecho Público de la Universidad del Zulia, pp 91-138.

MAINWARING, Scott y Matthew Shugart (1997): "Conclusion: Presidentialism and the Party System". En Scott Mainwaring y Matthew Shugart (Eds) Presidentialism and Democracy in Latin America. Cambridge: Cambridge University Press.

MAINWARING, Scott y Timothy Scully (1995): "Introduction: Party Systems in Latin America”. En Scott Mainwaring y Timothy Scully (Eds) Building Democratic Institutions. Party Systems in Latin America. Stanford, California: Stanford University Press.

Molina, José (1991): El Sistema electoral venezolano y sus consecuencias políticas. Valencia, Venezuela. Hermanos Vadell Editores-Instituto Interamericano de Derechos Humanos.

Molina, José y Carmen PÉrez (1994): “Venezuela: ¿Hacia un nuevo sistema de partidos? Las elecciones de 1993". Cuestiones Politicas, 13. Maracaibo: Instituto de Estudios Políticos y Derecho Público de la Universidad del Zulia, pp 63-90.

Molina, José y Carmen Pérez (1995): "Venezuela. Elecciones estatales y municipales 3 de diciembre de 1995". Boletin Electoral Latinoamericano XIV, julio-diciembre, pp. 45-63. 
Movimiento V RepúBlica (1998): La propuesta de Hugo Chávez para transformar a Venezuela. Caracas: Movimiento V República.

NoHLEn, Dieter (1994): Sistemas Electorales y Partidos Políticos. México: UNAM/Fondo de Cultura Económica.

Pedersen, Mogens (1983): “Changing Patterns of Electoral Volatility in European Party Systems, 1948-1977: Explorations in Explanations". En Hans Daalder y Peter Mair (Eds.): Western European Party Systems: Continuity and Change. Beverly Hills, California: Sage.

PÉrez B., Carmen (1992): "La hipótesis del elector flotante. El caso del Distrito Sucre del Estado Miranda”.
Cuestiones Políticas, 9. Maracaibo: Instituto de Estudios Políticos y Derecho Público de la Universidad del Zulia, pp. 57-69.

SäRLVIK, Bo y Ivor Crewe (1983): Decade of dealignment. The Conservative victory of 1979 and electoral trends in the 1970s. Cambridge: Cambridge University Press.

ShugarT, Matthew y John Carey (1992): Presidents and Assemblies. Constitutional Design and Electoral Dynamics. Cambridge: Cambridge University Press.

TORRES, Arístides (1985): "Fe y Desencanto Democrático en Venezuela”. Nueva Sociedad, 77, Mayo-Junio.

\section{RESUMEN}

El panorama político venezolano está sufriendo importantes cambios. El electorado ha roto con la estática estabilidad bipartidista que se logró en el llamado Pacto de Punto Fijo en 1958. En el artículo se exponen los antecedentes de orden político y económico de la transformación que se está desarrollando actualmente en la democracia venezolana. Se trata de un cambio que se ha mostrado a nivel electoral, donde nuevas fuerzas políticas sustituyen a los partidos tradicionales. La población ha perdido la confianza en los viejos partidos -Acción Democrática y COPEI- y las lealtades partidistas han desaparecido en favor de la personalización de la política y de la volatilidad del voto.

En el artículo se describen los cambios en el sistema electoral y sus consecuencias; los partidos políticos y sus candidatos; el fenómeno de la abstención; los resultados de las elecciones para el Congreso de la República, y el proceso de elección presidencial.

Palabras clave: Venezuela, elecciones, revolución democrática, Movimiento V República, Hugo Chávez Frías.

\section{ABSTRACT}

The political life in Venezuela is suffering important changes. The electorate has broken the static two-party stability achieved in 1958 with the so called Pacto de Punto Fijo. The article presents the political and economic background prior to the transformation that is actually taking place in the Venezuelan democracy.

The change was manifest at the electoral level, where new political forces are substituting the traditional parties. The population has lost confidence in the old parties - Acción Democrática y COPEI - and party loyalties have disappeared in favor of the personalization of politics and the volatility of the vote.

The article describes the changes in the electoral system and their consequences; political parties and their candidates; the phenomenon of abstention; the results of Congress elections and the process of presidential election.

Key words: Venezuela, elections, democratic revolution, Fifth Republic Movement, Hugo Chávez Frías. 